\title{
Structure, Solution Aggregation and UV Curing of Hyperbranched Poly(ester-amine)s with Terminal Acrylate Groups
}

\author{
Li-Ming TANG, ${ }^{\dagger}$ Yu FANG, and Ji FENG \\ Department of Chemical Engineering, Tsinghua University, Beijing 100084, P. R. China
}

(Received September 21, 2004; Accepted December 15, 2004; Published April 15, 2005)

\begin{abstract}
A series of hyperbranched poly(ester-amine)s (defined as HPEA-1-HPEA-5) with terminal acrylate groups were synthesized through Michael addition between piperazine $\left(\mathrm{A}_{2}\right)$ and trimethylolpropanetriacrylate (TMPTA) $\left(\mathrm{B}_{3}\right)$ under molar ratios ranged from $1 / 2$ to $1 / 1.08$. As increasing the molar ratio between piperazine and TMPTA, the degree of branching (DB), number average molecular weight $\left(M_{\mathrm{n}}\right)$, polydispersity index $\left(M_{\mathrm{w}} / M_{\mathrm{n}}\right)$ and glass transition temperature $\left(T_{\mathrm{g}}\right)$ of the polymers increased accordingly. Due to their amphiphilic flexible structures, the polymers could form stable aggregates in acetone-acidic water selective solution at volume ratio of $1 / 25$. The influences of polymer structures and the solution $\mathrm{pH}$ on the size and size distribution of the aggregates were measured by dynamic light scattering (DLS). The results indicated that smaller size was obtained for the polymer with higher $M_{\mathrm{n}}$, while the size distribution changed in accordance with $M_{\mathrm{w}} / M_{\mathrm{n}}$. At lower solution $\mathrm{pH}$, both smaller size and narrower size distribution were obtained. Furthermore, the polymer dispersions were irradiated by UV light to initiate polymerization of acrylate groups within the aggregates. The resulting cured particles possessed decreased size compared with the size of the corresponding aggregates as determined by DLS and transmission electron microscopy (TEM).

[DOI 10.1295/polymj.37.255]

KEY WORDS Hyperbranched Poly(ester-amine) / Amphiphilic Structure / Aggregation / UV Curing / Size and Size Distribution /
\end{abstract}

Dendritic polymers, including dendrimers and hyperbranched polymers have gained increasing interests during the past decade due to their unique physical properties. ${ }^{1}$ With well-defined molecular weight and architecture, dendrimers have been explored extensively as building blocks to construct supramolecular assemblies based on their catalyst, binding and optical properties. ${ }^{2}$ Researches on amphiphilic dendrimers consisting of polar core and apolar shell demonstrated that they could form inverted unimolecular micelles in solutions. ${ }^{3}$ Which makes them useful candidates as drug delivery carrier, ${ }^{4}$ photosensitive materials, ${ }^{5}$ liquid-liquid extractants, ${ }^{6}$ molecular nanocapsules ${ }^{7}$ and so on. However, the difficulty in their synthesis is now a great challenge for practical application.

Hyperbranched polymers resemble dendrimers in many aspects, such as high solubility, low solution and melt viscosities, lack of entanglement and so on. However, hyperbranched polymers can be prepared even through one-pot procedure and thus they have been considered as suitable substitutes for dendrimers in many aspects, including supramolecular assembly. ${ }^{8}$ Similar to dendrimers, amphiphilic hyperbranched polymers with surface hydrophobic alkyl chains and interior hydrophilic core could trap polar guest molecules within the molecules ${ }^{9}$ and form organized monolayer at air-water interfacial. ${ }^{10}$ Pyrene- labeled hyperbranched amphiphiles could form micelles in aqueous solution. ${ }^{11}$ We also observed hyperbranched polyester with hydrophilic shell and hydrophobic core could form global aggregates in selective solution. ${ }^{12}$ However, the influence of polymer structure on the aggregation behavior in solution has not been systematically investigated.

In this paper, a series of amphiphilic hyperbranched poly(ester-amine)s (defined as HPEA-1-HPEA-5) with terminal acrylate groups were synthesized and characterized. These polymers could form stable aggregates in acetone and acidic water selective solution. The influence of polymer structures and the solution $\mathrm{pH}$ on the size and size distribution of the aggregates were studied by dynamic light scattering (DLS). The dispersion was further irradiated by UV light to form cured particles through polymerization of acrylate groups within the aggregates.

\section{EXPERIMENTAL}

\section{Synthesis of Hyperbranched Poly(ester-amine)s}

Hyperbranched poly(ester-amine)s were prepared through Michael addition between piperazine $\left(\mathrm{A}_{2}\right)$ and trimethylolpropanetriacrylate (TMPTA) $\left(\mathrm{B}_{3}\right)$. For example, at piperazine/TMPTA molar ratio of $1 / 1.08$, the reaction procedure for HPEA-5 is described as

${ }^{\dagger}$ To whom correspondence should be addressed (E-mail: tanglm@mail.tsinghua.edu.cn). 


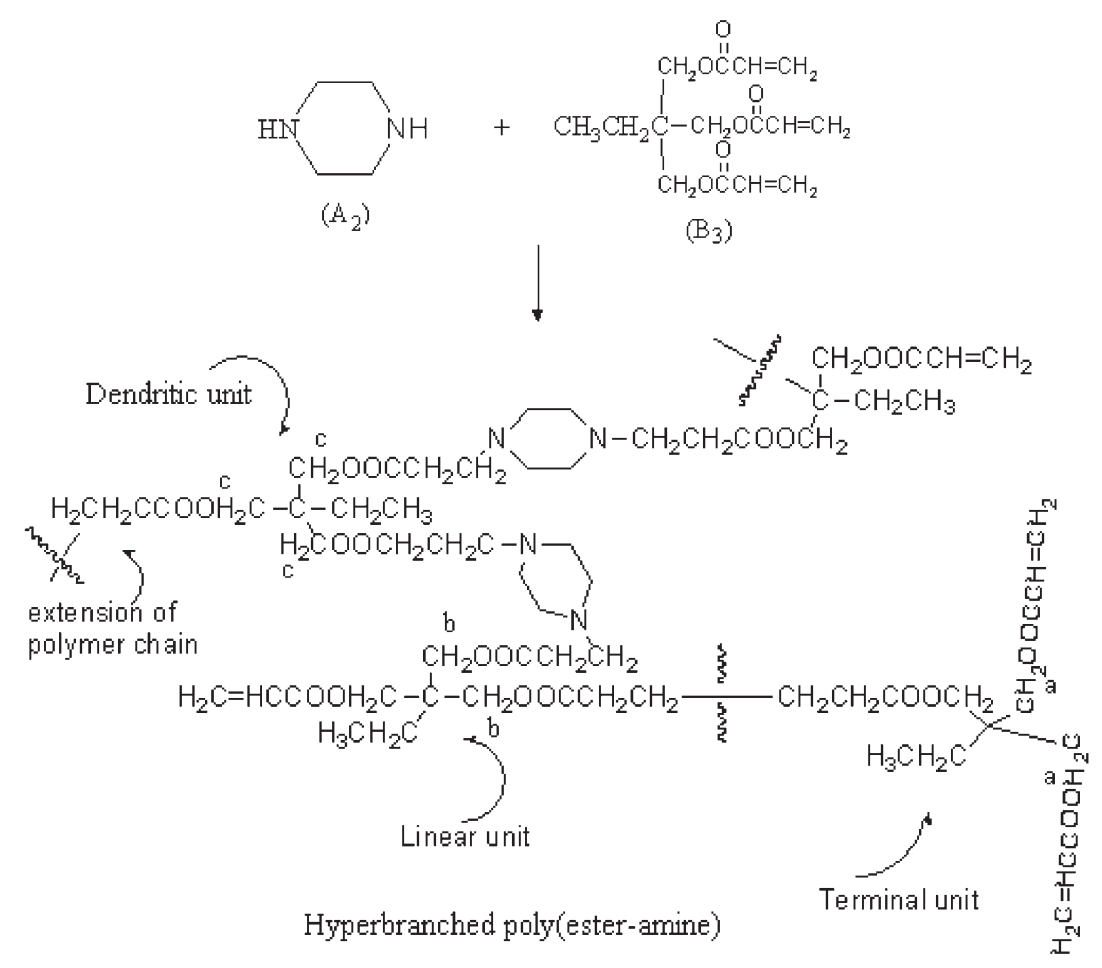

Figure 1. Synthetic procedure and structure of hyperbranched poly(ester-amine).

follows: $2.901 \mathrm{~g}(0.0098 \mathrm{~mol})$ TMPTA and $0.5 \mathrm{wt} \%$ hydroquinone as inhibitor were dissolved in $20 \mathrm{~mL}$ THF. $0.783 \mathrm{~g}(0.0091 \mathrm{~mol})$ piperazine was dissolved in $1 \mathrm{~mL}$ methanol and the solution was diluted by $20 \mathrm{~mL}$ THF. After dropping TMPTA solution into piperazine solution under ice bath within $1 \mathrm{~h}$, the temperature was slowly elevated to $30^{\circ} \mathrm{C}$ and the reaction was maintained for $48 \mathrm{~h}$. After removing the solvent by vacuum distillation, the crude product was washed twice by ether and dried to a constant weight in an oven under vacuum, thus to yield $3.198 \mathrm{~g}$ viscous yellow product in $86.8 \%$ yield. By the same procedure, the other polymers termed as HPEA-1, HPEA-2, HPEA-3 and HPEA-4 were prepared in 79.5, 82.1, 81.0 and $85.2 \%$ yields at piperazine/TMPTA molar ratio of $1 / 2.00,1 / 1.67,1 / 1.43$ and $1 / 1.20$, respectively. ${ }^{1} \mathrm{H}$ NMR $\left(300 \mathrm{MHz}, \mathrm{CDCl}_{3}\right): \delta \quad 0.85-0.92(\mathrm{~m}$, $\left.\mathrm{CH}_{3}-\right), 1.42-1.52\left(\mathrm{~m}, \mathrm{CH}_{3} \mathrm{CH}_{2}-\right), 2.47-2.53\left(\mathrm{~m}, \mathrm{R}^{\prime}-\right.$ $\left.\mathrm{OOCCH}_{2} \mathrm{CH}_{2} \mathrm{~N}\left(\mathrm{CH}_{2} \mathrm{R}\right)_{2}\right), 2.62-2.65$ (m, R'-OOC$\mathrm{CH}_{2} \mathrm{CH}_{2} \mathrm{NR}_{2}$ ), 4.04-4.18 (m, $\left.\mathrm{RCH}_{2} \mathrm{OCOR}^{\prime}\right)$, 5.92$6.37\left(\mathrm{~m},-\mathrm{COOC} H=\mathrm{CH}_{2}\right)$. FT-IR $(\mathrm{KBr}): \gamma\left(\mathrm{cm}^{-1}\right)=$ 2800-3000 (C-H sat.), 1730 (C=O), 1660 (conjugated $\mathrm{C}=\mathrm{C}), 1050(\mathrm{C}-\mathrm{N}$ stretch$)$.

\section{Formation of Aggregates and UV Curing}

Polymer of $0.1 \mathrm{~g}$ was dissolved in $4 \mathrm{~mL}$ acetone with or without $5 \mathrm{wt} \%$ benzophenone. The solution was mixed with $100 \mathrm{~mL}$ acidic deionized water for 15 min under ultrasonication. After maintained at room temperature for $12 \mathrm{~h}$, a stable milk-like dispersion was formed. The dispersion containing benzophenone was directly irradiated with a $1000 \mathrm{~W}$ medium pressure mercury arc lamp for 3 min to complete the curing reaction.

\section{Measurement}

${ }^{1} \mathrm{H}$ NMR spectra were recorded using Unity UNITY-200 spectrometer. FT-IR spectra were recorded on NICOLET 560 FT-IR spectrometer. GPC was performed in THF at ambient temperature using a column from Waters and was calibrated with polystyrene standards. Differentials scanning calorimetric (DSC) measurements were carried out using DuPont-2400 DSC Plus module. The polymer dispersion was measured by dynamic light scattering (DLS) using Malvern Zetasizer $3000 \mathrm{HS}$ instrument at an angle of $90^{\circ}$. The dispersion containing benzophenone was directly placed on a carbon-coated grid and imaged on a transmission electron microscopy (TEM, H-800).

\section{RESULTS AND DISCUSSION}

\section{Synthesis and Characterization}

Five hyperbranched poly(ester-amine)s (defined as HPEA-1-HPEA-5) were synthesized through Michael addition of piperazine $\left(\mathrm{A}_{2}\right)$ and TMPTA $\left(\mathrm{B}_{3}\right)$ under various molar ratios ranged from $1 / 2$ to $1 / 1.08$. The synthetic procedure was outlined in Figure 1, where the resulting polymer contains three structural units: (1) dendritic unit $\left(N_{\mathrm{d}}\right)$, (2) linear unit $\left(N_{\mathrm{L}}\right)$ and (3) terminal unit $\left(N_{\mathrm{t}}\right)$. It has been well known that direct polycondensation of $\mathrm{A}_{2}$ kind and $\mathrm{B}_{3}$ kind monomers 
generally results in gelation. ${ }^{8}$ In this study, gelated products were formed at short reaction time or high monomer concentration. If the total monomer concentration was lower than $10 \mathrm{wt} \%$ and the reaction was lasted for at least $48 \mathrm{~h}$ to consume all secondary amines in the system, soluble products could be prepared successfully.

The structure of HPEA was confirmed by FT-IR and ${ }^{1} \mathrm{H}$ NMR measurements. In the ${ }^{1} \mathrm{H}$ NMR spectra of the products, the peak (at $2.87 \mathrm{ppm}$ ) responding to $\mathrm{CH}_{2}$ next to secondary amine group is not observed. In order to calculate the degree of branching (DB), the multi-peaks at $3.80-4.30 \mathrm{ppm}$ in the ${ }^{1} \mathrm{H}$ NMR spectra for various samples were compared in Figure 2. It is clear that the peaks for the protons in $-\mathrm{CH}_{2} \mathrm{OOC}-$ move to higher frequency as more acrylate groups in TMPTA reacting with secondary amines. Through comparing the integral of each peak to that of the peaks within $0.85-0.98 \mathrm{ppm}$ (belong to $-\mathrm{CH}_{3}$ ), the
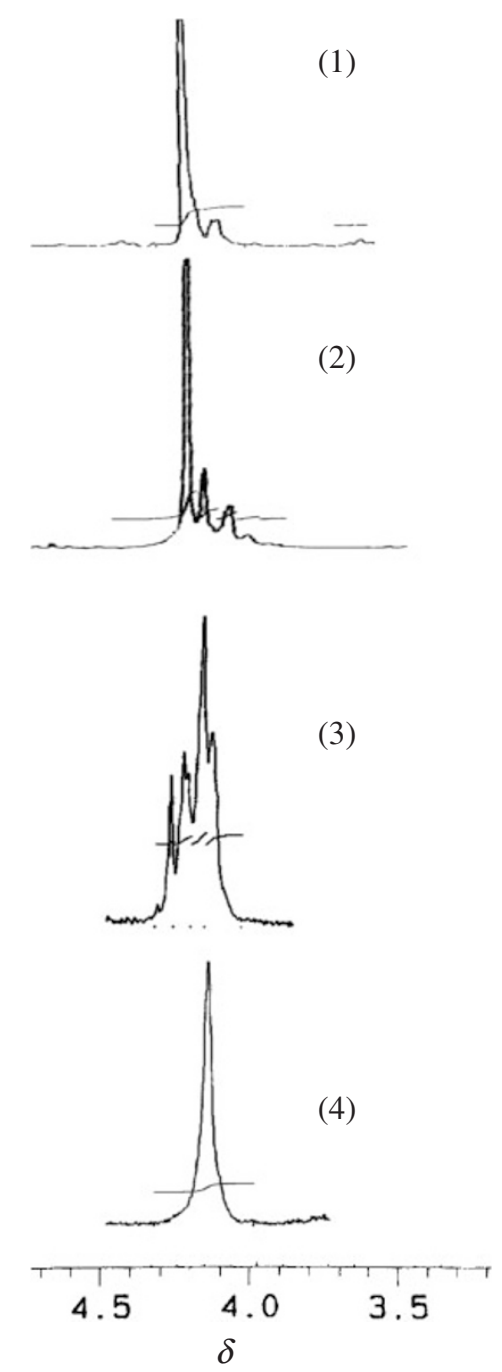

Figure 2. Comparing the peaks within $3.80-4.30 \mathrm{ppm}$ in the ${ }^{1} \mathrm{H}$ NMR spectra of various samples. Piperazine/TMPTA molar ratio: (1) $0 / 1$; (2) $1 / 8$; (3) $1 / 1.08$; (4) $1 / 0.33$. assignment of these peaks was assured. Based on this analysis, the peaks at 4.14-4.16, 4.05-4.08 and 4.02$4.05 \mathrm{ppm}$ were attributed to the protons of $\mathrm{a}(\mathrm{H})$ in terminal unit, $b(\mathrm{H})$ in linear unit and $\mathrm{c}(\mathrm{H})$ in dendritic unit (see Figure 1), respectively. In order to prove this result, model reactions between piperidine and TMPTA at molar ratios of $1 / 1,2 / 1$ and $3 / 1$ were further carried out by a procedure similar to the reaction between piperazine and TMPTA. The peaks at 4.00$4.20 \mathrm{ppm}$ for ${ }^{1} \mathrm{H}$ NMR spectra of TMPTA and the resulting samples were shown in Figure 3. Based on the structures of model compounds and the integral of the peaks, the assignment of the peaks were defined in Figure 3. It is obvious that the result is the same as that obtained from Figure 2.

Based on the integration of corresponding peaks of the ${ }^{1} \mathrm{H}$ NMR spectra, the relative contents of the structural units were calculated. From the results in Table I, it is noticed that $N_{\mathrm{t}}$ decreased, while both $N_{\mathrm{d}}$ and $N_{\mathrm{L}}$ increased with improving piperazine/ TMPTA molar ratio. In addition, $N_{\mathrm{t}}$ is higher than $N_{\mathrm{d}}$ for the first three samples, but is lower than $N_{\mathrm{d}}$ for the last two samples. It may suggest that some cyclized structures were formed in HPEA-4 and HPEA5. The values of $\mathrm{DB}$ for the polymers calculated by Frey's equation, ${ }^{13} \mathrm{DB}=2 N_{\mathrm{d}} /\left(N_{\mathrm{L}}+2 N_{\mathrm{d}}\right)$, were summarized in Table I. Since $N_{\mathrm{d}}$ is a major parameter for calculating $\mathrm{DB}$, the values of $\mathrm{DB}$ increased from HPEA-1 to HPEA-5.

The polymers were further characterized by GPC and DSC measurements, and the results were shown in Table I. At higher piperazine/TMPTA molar ratio, more acrylate groups could react with secondary amines, thus resulted in a polymer with higher number average molecular weight $\left(M_{\mathrm{n}}\right)$. Because the reaction proceeded in a random fashion, as increasing in molecular weight, the molecular structure turned to be more irregular and thus the polymer had an increased polydispersity index $\left(M_{\mathrm{w}} / M_{\mathrm{n}}\right)$. Furthermore, the higher glass transition temperature $\left(T_{\mathrm{g}}\right)$ obtained at higher piperazine/TMPTA molar ratio should be attributed to the lower moving ability of the chains restricted by branching structures. All the polymers possess $T_{\mathrm{g}}$ lower than $0^{\circ} \mathrm{C}$, indicating of their highly flexible chains.

\section{Aggregation Behavior in Selective Solution}

Despite of structural difference, all the five polymers in Table I were dissolve completely in organic solvents, such as methanol, THF and acetone, but not in pure water. If the polymers were directly dispersed into acidic aqueous solution, the rapid association of exterior hydrophobic acrylate groups can prevent interior tertiary amine groups from ionizing, thus only a partly of the polymers was dissolved in 
<smiles>C=C(C)OC(=O)CC(CC)(CC)COC(=O)CC</smiles><smiles>C=C(C)OCC(CC)(CC)CC(=O)OCCN1CCCCC1</smiles>

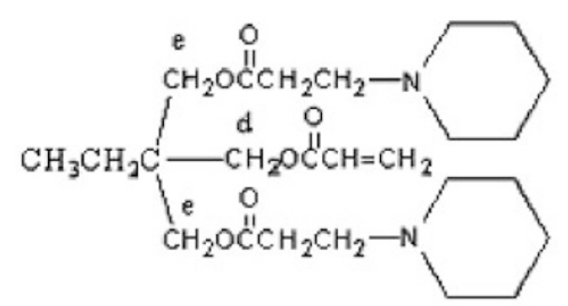<smiles>CCC1(CC)CC(CC(=O)OCCN2CCCCC2)C(OC(=O)CN2CCCCC2)C1</smiles>
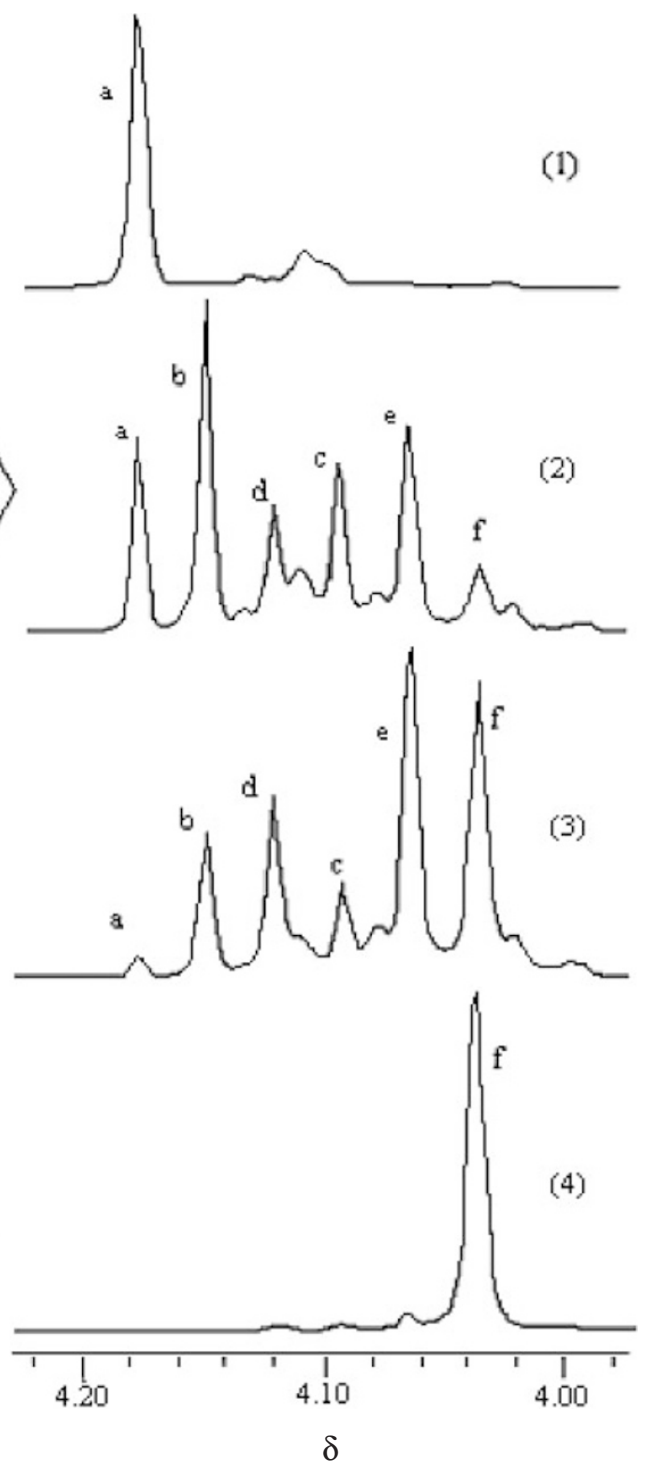

Figure 3. Comparing the peaks within 4.00-4.20 ppm in the ${ }^{1} \mathrm{H}$ NMR spectra of model samples prepared from piperidine and TMPTA. Piperidine/TMPTA molar ratio: (1) $0 / 1$; (2) $1 / 1$; (3) $2 / 1$; (4) $3 / 1$.

Table I. Structural analysis and $T_{\mathrm{g}}$ for the polymers

\begin{tabular}{ccccccccc}
\hline \multirow{2}{*}{ Sample } & $\begin{array}{c}\text { Piperazine/TMPTA } \\
\text { /molar ratio }\end{array}$ & \multicolumn{3}{c}{ Structure unit/\% } & \multirow{2}{*}{ DB $/ \%$} & \multirow{2}{*}{$M_{\mathrm{n}}$} & \multirow{2}{*}{$M_{\mathrm{w}} / M_{\mathrm{n}}$} & \multirow{2}{*}{$T_{\mathrm{g}} /{ }^{\circ} \mathrm{C}$} \\
\cline { 2 - 5 } & $N_{\mathrm{t}}$ & $N_{\mathrm{L}}$ & \multicolumn{1}{c}{$N_{\mathrm{d}}$} & & & & \\
\hline HPEA-1 & $1 / 2.00$ & 50.5 & 41.2 & 8.3 & 28.7 & 1818 & 1.325 & -42.5 \\
HPEA-2 & $1 / 1.67$ & 48.3 & 36.5 & 15.2 & 45.4 & 1948 & 1.347 & -32.4 \\
HPEA-3 & $1 / 1.43$ & 37.3 & 44.0 & 18.7 & 45.9 & 2222 & 1.560 & -21.2 \\
HPEA-4 & $1 / 1.20$ & 22.0 & 53.3 & 24.7 & 48.1 & 2546 & 1.842 & -11.4 \\
HPEA-5 & $1 / 1.08$ & 14.2 & 55.3 & 30.5 & 52.5 & 3246 & 3.754 & -9.3 \\
\hline
\end{tabular}

the solution, especially for the one with more terminal acrylate groups.

Since acetone is a good solvent for the whole molecule and acidic water is a good solvent for only the interior part with ionizable tertiary amines, acetoneacidic water mixture could be regarded as a selective solvent for the polymers. If the polymers in a small amount of acetone were mixed with large amount of acidic water at volume ratio of $1 / 25$, stable translucent milk-like dispersions were resulted, indicating the formation of intermolecular aggregates in the systems. In acetone-acidic water selective solution, under the help of acetone, the acrylate groups could pack together to form a hydrophobic tail due to the flexibly chains of the molecule. Meanwhile, the core turned outside and reacted with hydrogen protons, 
Structure and Aggregation Behaviors of Hyperbranched Polymers

Table II. Size and size distribution for the aggregates in selective solution

\begin{tabular}{|c|c|c|c|c|c|c|}
\hline \multirow{2}{*}{ Sample } & \multicolumn{2}{|c|}{ Amount of groups in 100 units } & \multirow{2}{*}{$D_{\mathrm{z}} / \mathrm{nm}$} & \multirow{2}{*}{$D_{\mathrm{v}} / \mathrm{nm}$} & \multirow{2}{*}{$D_{\mathrm{n}} / \mathrm{nm}$} & \multirow{2}{*}{$D_{\mathrm{v}} / D_{\mathrm{n}}$} \\
\hline & Tertiary amine & Acrylate group & & & & \\
\hline HPEA-1 & 157.8 & 142.2 & 306.0 & 386.3 & 272.0 & 1.42 \\
\hline HPEA-2 & 166.9 & 133.1 & 267.5 & 344.5 & 215.5 & 1.60 \\
\hline HPEA-3 & 181.4 & 118.6 & 237.8 & 293.2 & 176.0 & 1.67 \\
\hline HPEA-4 & 202.7 & 97.3 & 219.3 & 248.8 & 141.3 & 1.76 \\
\hline HPEA-5 & 216.3 & 83.7 & 241.4 & 96.5 & 27.4 & 3.52 \\
\hline
\end{tabular}

Conditions: polymer: $0.1 \mathrm{~g}$, acetone: $4 \mathrm{~mL}$, water: $100 \mathrm{~mL}$, solution $\mathrm{pH}: 5.0$

thus to form a hydrophilic head. Through hydrophobic association, many such head-tail amphiphilic molecules could further self-assemble into aggregates stabilized by double electrical layers. So the aggregate should be composed of a hydrophilic shell and a hydrophobic core.

Because the aggregates formed in solutions were in sphere shapes as observed by TEM, the particle size and size distribution were determined by DSL measurements. The number average size defined as $D_{\mathrm{n}}=$ $\Sigma d_{\mathrm{i}} / n_{\mathrm{i}}$ is arithmetical average. The volume average size defined as $D_{\mathrm{v}}=\left(\Sigma n_{\mathrm{i}} d_{\mathrm{i}}^{3} / \Sigma n_{\mathrm{i}}\right)^{1 / 3}$ could be considered as weight average size if the particle density is constant. $\mathrm{Z}$ average size $D_{\mathrm{z}}$ is based on the measurement of light scattering intension and is an average of accumulative total.

According to the molecular structure of the polymers, there are one tertiary amine and two acrylate groups in terminal unit, two amines and one acrylate in linear unit, and only three tertiary amines in dendritic units, the numbers of tertiary amines and acrylates groups in 100 structural units of the polymers could be calculated based on the content of the structural units listed in Table I. From the results in Table II, the content of tertiary amines increased and the content of acrylate groups decreased from HPEA-1 to HPEA-5. Due to the different contents in ionizable tertiary amine and hydrophobic acrylate groups, the polymers demonstrate different amphiphilic features and aggregation behaviors in acetone-acidic water selective solution.

The polymers in Table I were dispersed into acetone-acidic water selective solution at $\mathrm{pH} \mathrm{5.0} \mathrm{and}$ the aggregate size and size distribution were determined by DSL. Comparing $D_{\mathrm{v}}$ and $D_{\mathrm{v}} / D_{\mathrm{n}}$ in Table II with $M_{\mathrm{n}}$ and $M_{\mathrm{w}} / M_{\mathrm{n}}$ in Table I, respectively, it is interesting to note that smaller size is obtained at higher $M_{\mathrm{n}}$, while $D_{\mathrm{v}} / D_{\mathrm{n}}$ changes in accordance with $M_{\mathrm{w}} / M_{\mathrm{n}}$. According to the contents of tertiary amines and acrylate groups in the molecules, the polymer with lower molecular weight has less tertiary amine groups and more hydrophobic acrylate groups and thus is more hydrophobic. In selective solution, to get a hydrophilic-hydrophobic balance, it is needed for more mole-
Table III. Influence of solution $\mathrm{pH}$ on the size of the aggregates

\begin{tabular}{cccccccc}
\hline \multirow{2}{*}{ Solution $\mathrm{pH}$} & \multicolumn{3}{c}{ HPEA-2 } & & \multicolumn{3}{c}{ HPEA-4 } \\
\cline { 2 - 4 } \cline { 6 - 8 } & 2.8 & 3.6 & 5.0 & & 3.6 & 5.0 & 6.2 \\
\hline$D_{\mathrm{z}} / \mathrm{nm}$ & 235.4 & 255.2 & 281.4 & & 151.5 & 209.3 & 218.9 \\
$D_{\mathrm{v}} / \mathrm{nm}$ & 205.2 & 338.0 & 392.0 & & 124.0 & 185.3 & 257.0 \\
\hline
\end{tabular}

cules to participate in aggregation to increase surface static repulsive force and result in somewhat bigger aggregates. On the contrary, the one with higher molecular weight is more hydrophilic and contains more dendritic units. In this case, the molecules are difficult to associate with each other and smaller aggregates are formed. In addition, the polymer with higher $M_{\mathrm{w}} / M_{\mathrm{n}}$ possesses more irregular molecular structure. The intermolecular aggregation tends to proceed in more irregular manner, thus result in a wider size distribution for the aggregates.

Based on HPEA-2 and HPEA-4, the influence of solution $\mathrm{pH}$ on aggregate size and size distribution were further investigated. From the results in Table III, the aggregate size became large as increasing solution $\mathrm{pH}$ in both HPEA-2 and HPEA-4 systems, which could be attributed to the decrease in ionizing degree of tertiary amines. In addition, the size for HPEA-4 is larger than that for HPEA-2 under the same $\mathrm{pH}$ due to their different amphiphilic structures. The distribution of $D_{\mathrm{v}}$ for HPEA-2 and HPEA4 was shown in Figure 4 and Figure 5, respectively. From Figure 4, the particle size concentrated at around $200 \mathrm{~nm}$ at $\mathrm{pH} 2.8$. As increasing the $\mathrm{pH}$, the size distribution became wider, the peak at $200 \mathrm{~nm}$ became small and the peak at $400 \mathrm{~nm}$ became large gradually, which is in accordance with the results in Table III. From Figure 5, HPEA-4 possessed a wider size distribution than that for HPEA-2 which should be attributed to the more irregular molecular architecture of HPEA-4.

\section{UV Radiation of the Dispersion}

Due to the existence of some terminal acrylate groups in the molecules, the polymers could be re- 


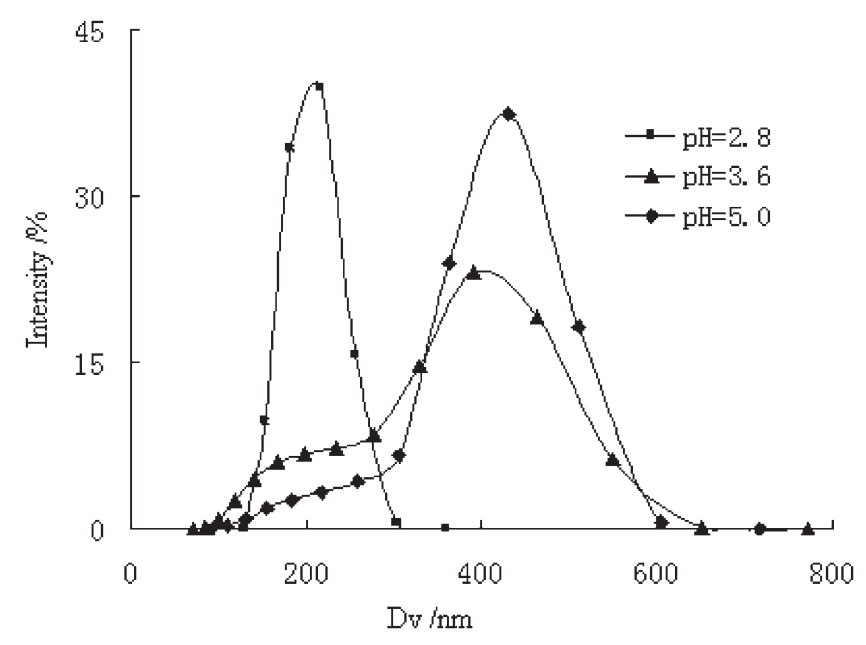

Figure 4. Influence of solution $\mathrm{pH}$ on the distribution of $D_{\mathrm{v}}$ in HPEA-2 system.

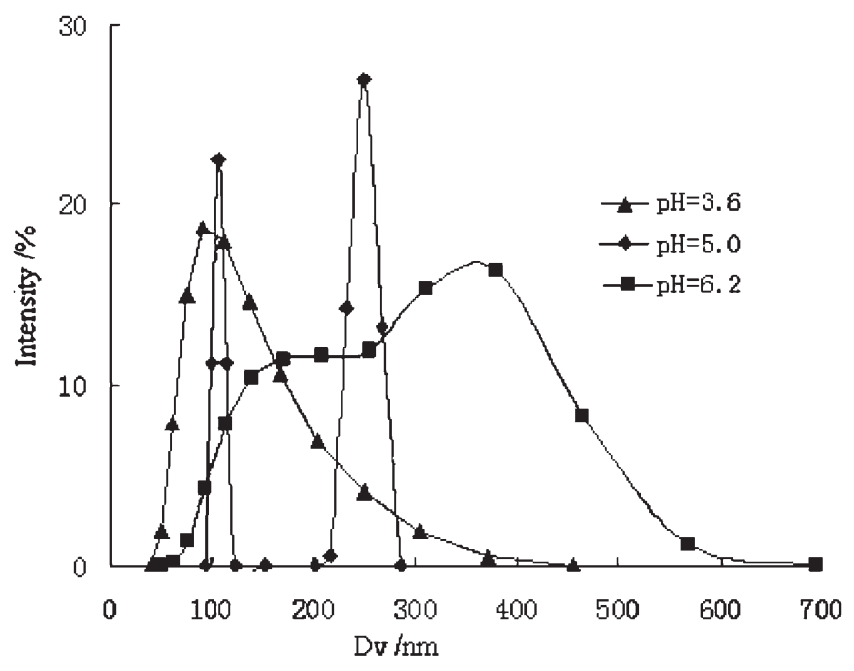

Figure 5. Influence of solution $\mathrm{pH}$ on the distribution of $D_{\mathrm{v}}$ in HPEA-4 system.

Table IV. Effect of solution $\mathrm{pH}$ on the size of cured particles

\begin{tabular}{cccccccc}
\hline \multirow{2}{*}{ Solution $\mathrm{pH}$} & \multicolumn{3}{c}{ HPEA-2 } & & \multicolumn{3}{c}{ HPEA-4 } \\
\cline { 2 - 4 } \cline { 6 - 8 } & 2.8 & 3.6 & 5.0 & & 3.6 & \multicolumn{1}{c}{5.0} & 6.2 \\
\hline$D_{\mathrm{z}} / \mathrm{nm}$ & 197.2 & 197.0 & 218.8 & & 127.9 & 145.9 & 146.9 \\
$D_{\mathrm{v}} / \mathrm{nm}$ & 188.8 & 249.3 & 287.0 & & 80.8 & 82.2 & 102.6 \\
\hline
\end{tabular}

Conditions: polymer: $0.1 \mathrm{~g}$, benzophenone: $5 \mathrm{wt} \%$, acetone: $4 \mathrm{~mL}$, water: $100 \mathrm{~mL}$, solution $\mathrm{pH}: 3.6$

garded as starting materials for cured samples, especially for cured particles in solution. If suitable amount of a common UV initiator, benzophenone, was dissolved in HPEA-2 and HPEA-4 acetone solution, aggregates were formed after mixing the solution with acidic water at volume ratio of $1 / 25$. Due to its hydrophobic nature, benzophenone can get into the interior of the aggregates and contact with acrylate groups. After irradiated by UV light, no precipitate

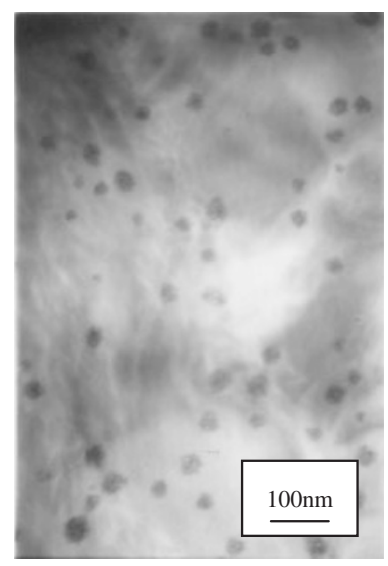

(1)

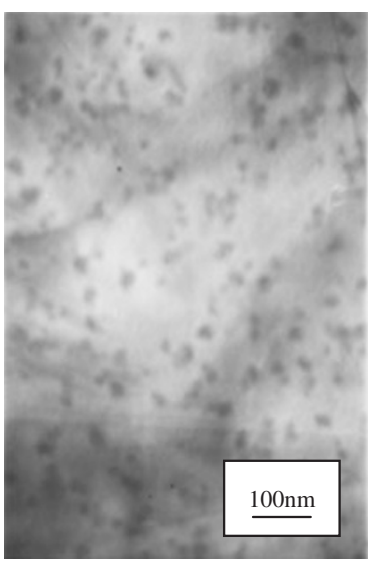

(2)
Figure 6. TEM photographs for the particles formed in selective solution.

was formed in the dispersion. The particles size measured by DSL was shown in Table IV. All the sizes of particle are smaller than those of the corresponding aggregates in Table III. The decrease in size could be attributed to the polymerization of acrylate groups initiated by UV initiator, which may cause the shrinkage of the particles. For HPEA-2 system at solution $\mathrm{pH}$ 2, fine hard powder was obtained after evaporation the solvent. DSC measurement of the cured sample indicated it possesses $T_{\mathrm{g}}$ of $138^{\circ} \mathrm{C}$, much higher than that of its prepolymer. It indicated rather compact networks had been formed within the particles. Its FT-IR spectrum showed the characteristic band for conjugated vinylic groups at $1660 \mathrm{~cm}^{-1}$ was completely disappeared. These results clearly indicated that cured particles were formed during the irradiation of the dispersion. The high reaction extent of acrylate groups further demonstrated they located mainly at the core part of the aggregates and reacted easily with each other.

The samples were further imagined by TEM. Since typical staining agents with heavy metal ions can influence the aggregation behavior of the molecules, benzophenone was used directly as staining agent for TEM samples. Figure 6 shows the TEM photographs for the particles formed in the selective solution before and after irradiation. In both cases, globular particles with diameter less than $60 \mathrm{~nm}$ were observed. After irradiation, the particle size become smaller and the size distribution become wider. However, the size observed by TEM was much smaller than that obtained by DSL. In selective solution, the aggregates were in swelling state with solvent embedded. In preparing TEM samples, the evaporation of solvent could not only make the aggregates shrinking, but also change the surrounding environment for the aggregates, for example solution $\mathrm{pH}$. So the particle 
size observed by TEM is different from that obtained by DSL. Nevertheless, TEM results could be used to define the size of drying particles.

From the above results, it is shown that amphiphilic hyperbranched polymers could form stable aggregates in selective solution and the aggregate sizes were controlled by polymer structures and solution $\mathrm{pH}$. Such aggregates have potential applications as hydrophobic drug capsule and $\mathrm{pH}$ controlled release system.

Acknowledgment. The financial support from NSFC under the projects 20204005, 50233030, and basic research fund of Tsinghua University, China JC 2002030 is gratefully acknowledged.

\section{REFERENCES}

1. O. A. Matthews, A. N. Shipway, and S. J. Fraser, Prog. Polym. Sci., 23, 1 (1998).

2. D. A. Tomalia and I. Majoros, J. Macromol. Sci., Polym. Rev., 43, 411 (2003).

3. A. P. H. J. Schenning, C. Elissen-Román, J.-W. Weener, M. W. P. L. Baars, S. J. van der Gaast, and E. W. Meijer,
J. Am. Chem. Soc., 120, 8199 (1998).

4. S. Fuchs, K. Timo, O. Henning, T. Schoneberg, P. Franke, R. Gust, and A. D. Schuter, Chem.-Eur. J., 10, 1167 (2004).

5. G. D. Zhang, N. Nishiyama, A. Harada, D. L. Jiang, T. Aida, and K. Kataoka, Macromolecules, 36, 1304 (2003).

6. M. W. P. L. Baars, P. E. Froehling, and E. W. Meijer, Chem. Commun., 1959 (1997).

7. S. Stevelmans, J. C. M. van Hest, J. F. G. A. Jansen, D. A. F. J. van Boxtel, E. M. M. de Brabande-van den Berg, and E. W. Meijer, J. Am. Chem. Soc., 118, 7398 (1996).

8. C. Gao and D. Yan, Prog. Polym. Sci., 29, 183 (2004).

9. A. Sunder, M. Krämer, R. Hanselmann, R. Muelhaupt, and H. Frey, Angew. Chem., Int. Ed., 38, 3552 (1999).

10. X. Zhai, S. Peleshanko, N. S. Klimenko, K. L. Genson, D. Vaknin, M. Ya. Vortman, V. V. Shevchenko, and V. V. Tsukruk, Macromolecules, 36, 3101 (2003).

11. C. Gao, D. Y. Yan, B. Zhang, and W. Chen, Langmuir, 18, 3708 (2002).

12. T. Qiu, L. M. Tang, Z. W. Fu, X. L. Tuo, D. S. Liu, and W. T. Yang, Polym. Adv. Technol., 15, 65 (2004).

13. D. Holter, A. Burgrath, and H. Frey, Acta Polym., 48, 30 (1997) 